\title{
Characterization of the Chalcone Synthase Genes Expressed in flowers of the Common and Japanese Morning Glories.
}

\author{
Yasuyo Johzuka-Hisatomi, Atsushi Hoshino, Tomoko Mori, \\ Yoshiki Habu and Shigeru Iida* \\ National Institute for Basic Biology, Myodaiji, Okazaki, \\ Aichi 444-8585, Japan
}

(Received 16 August 1999, accepted 20 September 1999)

\begin{abstract}
The $C H S$ genes encoding chalcone synthase for flavonoid biosynthesis in the common and Japanese morning glories comprise a multigene family. Among these Ipomoea CHS genes, the CHS-D gene is the most abundantly expressed in the pigmented young flower buds and is primarily responsible for flower pigmentation. Majority of the remaining $C H S$ transcripts in the flower buds are produced from the $C H S-E$ gene. We characterized the genomic DNA segments of these $C H S-D$ and $C H S-E$ genes. Both genes have two exons with identical intron positions and carry several copies of two mobile element-like sequences with short terminal inverted repeats, MELS3 and MELS6 of around 200-300 bp. Small tandem repeats were also found in these $C H S$ gene regions. The $C H S-D$ and $C H S-E$ genes are expressed predominantly in flower limbs and tubes, respectively. These structural and functional features and their evolutionary implications are discussed.
\end{abstract}

\section{INTRODUCTION}

Flavonoids are the major flower pigments in plants and also play important roles in many other biological functions, including protection against UV light, plant-microbe interactions, and male fertility (Harborne 1994, Shirley 1996). A key enzyme for flavonoid biosynthesis is chalcone synthase (CHS) which catalyzes the formation of naringenin chalcone (Martin 1993). The CHS genes comprise a multigene family in many species, although a few plants such as Antirrhinum and Arabidopsis are known to carry single copies of the gene (Harborne 1994). Durbin et al. (1995) have reported isolation of four $C H S$ genes, CHS-A, CHS-B, CHS-C and CHS-PS, from the common morning glory (Ipomoea purpurea or Pharbitis purpurea). These CHS genes are members of the Ipomoea CHS gene family, which is comprised of 13 CHS genes from 7 Ipomoea species, including the $C H S-A$ and $C H S-B$ genes of the Japanese morning glory (Ipomoea nil or Pharbitis nil). These Ipomoea CHS genes are distantly related to the other known $C H S$ sequences in a phylogenetic tree (Durbin et al., 1995).

During studies to characterize the mutable $a^{\text {flaked }}$ allele for flower variegation in the common morning glory (Iida et al., 1995, Hisatomi et al., 1997), we have isolated a new $C H S$ cDNA, named $C H S-D[\mathrm{M}]$, from young buds of pig-

\footnotetext{
* Corresponding author.
}

mented flowers (Habu et al., 1997). The symbol $[\mathrm{M}]$ is added to genes derived from the common morning glory and $[\mathrm{J}]$ to genes from the Japanese morning glory in order to avoid confusion (Hisatomi et al., 1997, Fukada-Tanaka et al., 1997). We also obtained another $C H S-E[\mathrm{M}] \mathrm{cDNA}$ from the morning glory and the corresponding $C H S$ cDNAs, $C H S-D[J]$ and $C H S-E[J]$, from pigmented flower buds of the Japanese morning glory (Fukada-Tanaka et al., 1997). Nucleotide and amino acid identities between the $C H S-D$ and $C H S-E$ cDNAs are about $86 \%$ and around $70 \%$, respectively, and they are more closely related to the other known $C H S$ sequences than the previously characterized Ipomoea CHS genes (Fukada-Tanaka et al., 1997). Among these $C H S$ genes, $C H S-D$ in both plants was found to be predominantly expressed in the pigmented buds and drastically reduced in the white flower buds. Indeed, the $C H S-D[\mathrm{M}]$ and $C H S-E[\mathrm{M}] \mathrm{cDNAs}$ were found to constitute approximately $85 \%$ and $15 \%$ of cDNAs obtained from young pigmented buds of the common morning glory, respectively, and less than $1 \%$ of cDNAs corresponded to the CHS-A[M] cDNAs (Fukada-Tanaka et al., 1997). Subsequently, we found that the mutable $a^{\text {flaked }}$ allele is caused by insertion of a new transposable element, Tip100, into the CHS-D $[\mathrm{M}]$ gene (Habu et al., 1998). Tip100 is $3.9 \mathrm{~kb}$ long and belongs to the Ac/Ds family. Since the plant carrying the mutable $a^{\text {flaked }}$ allele blooms white flowers with colored spots and sectors, the $C H S-D[\mathrm{M}]$ gene must be primarily responsible for colora- 
tion in these tissues. In this paper, we describe structural characteristics of the genomic sequences of the $C H S-D$ and $C H S-E$ genes in the common and Japanese morning glories and discuss their expression patterns in pigmented flowers.

\section{MATERIALS AND METHODS}

Plant lines. The line KK/FR-35 of the common morning glory bears red flowers, the lines KK/VR-37 and KK/VR40 carrying the mutable $a^{\text {flaked }}$ allele produce white flowers with red variegations, and line KK/WP-3 containing two copies of Tip 100 within the $C H S-D[\mathrm{M}]$ intron displays stable white flowers (Hisatomi et al., 1997, Habu et al., 1998). The line KK/SSB-4 of the Japanese morning glory carrying the mutable $a-3^{\text {flecked }}$ allele for flower variegation was described before (Inagaki et al., 1994). The mutable $a-3^{\text {flecked }}$ allele was caused by the insertion of Tpn1 within the $D F R-B[J]$ gene encoding dihydroflavonol 4-reductase (DFR) in the anthocyanin biosynthesis pathway.

Nucleic acids procedures. General methods for genomic cloning were according to Sambrook et al., (1989) and were modified as described before (Inagaki et al., 1994, Hisatomi et al., 1997). Characterization of the $C H S-D[\mathrm{M}], C H S-E[\mathrm{M}]$ and $C H S-D[J]$ cDNAs was described before (Fukada-Tanaka et al., 1997).

Using the CHS-D[M] cDNA as probe, a genomic $5.7 \mathrm{~kb}$ $E c o$ RI fragment containing the entire $C H S-D[\mathrm{M}]$ sequence from KK/FR-35 was cloned into the $\lambda$ ZAP Express vector (Stratagene). Since the $3.9 \mathrm{~kb}$ Tip100 contains two EcoRI sites inside the elements, the genomic $3.0 \mathrm{~kb}$ and $6.5 \mathrm{~kb}$ $E c o R I$ fragments containing the first and second exons of the CHS-D $[\mathrm{M}]$ gene together with the left and right terminal sequences of Tip100 from KK/VR-40 were also cloned into the same $\lambda$ vector. The plasmids containing the cloned fragments were prepared by in vivo excision from these $\lambda$ clones, and the cloned fragments were sequenced by the chain termination method (Sambrook et al., 1989).

Around $7.5 \mathrm{~kb} E c o$ RI fragment carrying the $C H S-E[\mathrm{M}]$ gene was cloned from KK/VR-40 using the CHS-E[M] cDNA, and about $3.1 \mathrm{~kb}$ of DNA containing the entire $C H S-E[\mathrm{M}]$ gene was sequenced (see Fig. 1). About $9.6 \mathrm{~kb}$ and $9.3 \mathrm{~kb} E c o R I$ fragments containing the entire $C H S$ $D[J]$ and $C H S-E[J]$ sequences were also cloned from KK/
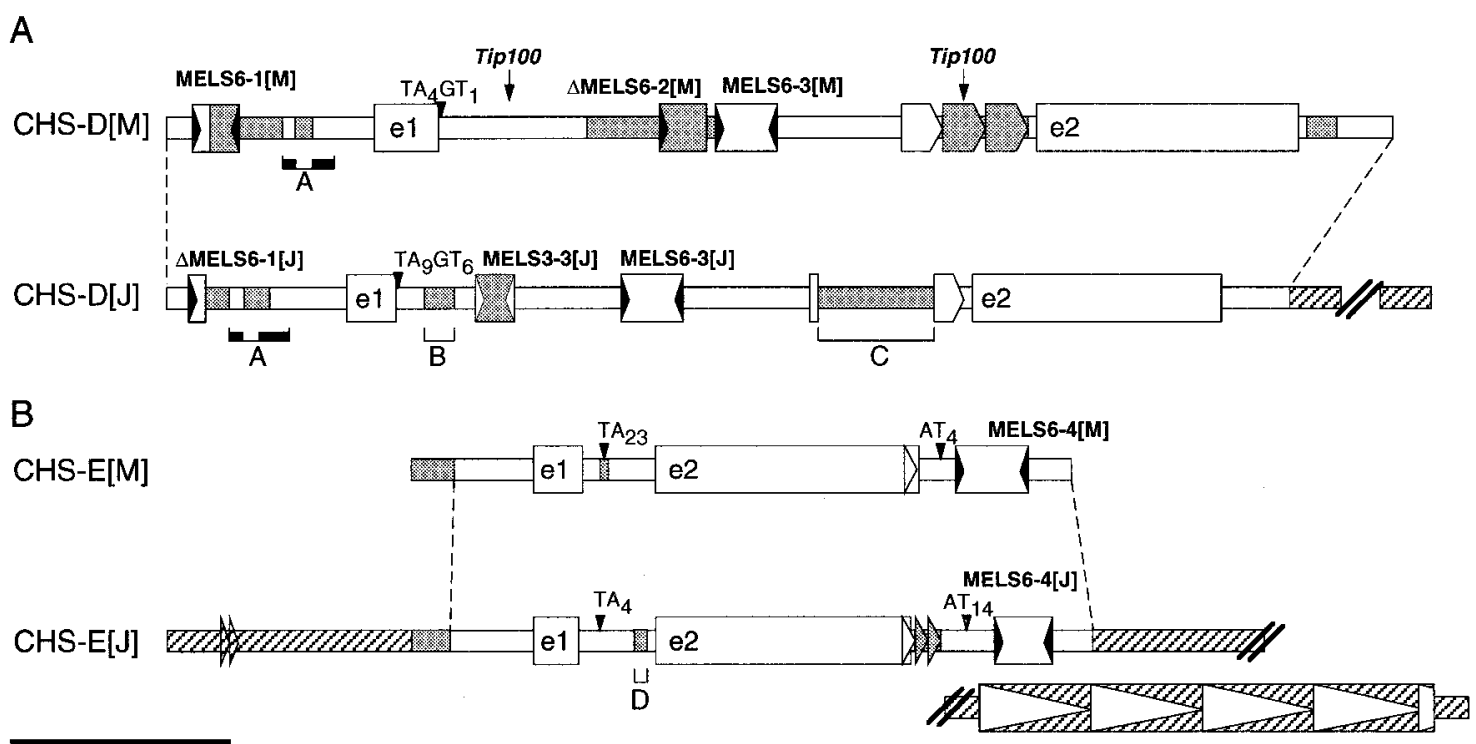

$1 \mathrm{~kb}$

Fig. 1. The genomic $C H S[\mathrm{M}]$ and $C H S[\mathrm{~J}]$ regions in the morning glories. Physical maps of the $C H S-D$ regions (A) and the $C H S-E$ regions (B). The horizontal boxes indicate the sequenced genomic CHS segments, and the hatched franking areas in the genomic boxes are unique regions in each sequenced segment. The open boxes with e 1 and e 2 indicate the $C H S$ exons deduced from the $C H S$ cDNA sequences (Fukada-Tanaka et al., 1997). The comparison of genomic sequences in these CHS regions was carried out by GeneWorks software (IntelliGenetics, Inc.). The open boxes within the genomic segments represent homologous regions between these sequenced segments whereas the shadowed parts indicate non-homologous areas. The MELS elements are represented by larger rectangles. The direct repeats in the $C H S-D[\mathrm{M}]$ intron and in the $C H S-E[\mathrm{~J}]$ region are indicated by the horizontal hexagonal and triangle arrowheads, respectively. The regions indicated by the brackets with A under the maps exhibit homology to the 3' DFR-A[M] terminal sequence, and the homologous regions are represented by the thick bars in the brackets. The regions indicated by the brackets $\mathrm{B}, \mathrm{C}$ and $\mathrm{D}$ are 134 bp, $529 \mathrm{bp}$ and $68 \mathrm{bp}$ long, and are flanked by $5 \mathrm{bp}, 2 \mathrm{bp}$ and $6 \mathrm{bp}$ direct repeats, respectively. The vertical arrows with Tip100 above the $C H S-D[\mathrm{M}]$ intron indicate the insertion sites of the transposable element Tip100 into the CHS-D [M] gene (Habu et al., 1998). The mutable $a^{\text {flaked }}$ lines KK/VR-37 and KK/VR-40 carry a single Tip100 at the 5' site whereas the stable white flower line KK/WP-3 contains an additional Tip100 at the 3' site. The small vertical arrows with $\mathrm{TA}_{4} \mathrm{GT}_{1}, \mathrm{TA}_{9} \mathrm{GT}_{6}, \mathrm{TA}_{23}, \mathrm{TA}_{4}, \mathrm{AT}_{4}$ and $\mathrm{AT}_{14}$ indicate the sites of the polymorphic microsatellite sequences, e.g. $\mathrm{TA}_{9} \mathrm{GT}_{6}$ represents 9 tandem TA repeats followed by 6 tandem GT repeats. 
SSB-4 into $\lambda$ DASH II (Stratagene) using the CHS-D $[\mathrm{J}]$ cDNA probe. The cloned fragments were subcloned into pHSG396 (TaKaRa BIOMEDICALS) and subsequently sequenced. The nucleotide sequences reported in this paper have been submitted to DDJB under the accession numbers AB004905 (CHS-D[M]), AB027533 (CHS-D[J]), AB027534 (CHS-E[M]), and AB027535 (CHS-E[J]).

\section{RESULTS AND DISCUSSION}

The genomic structures of the CHS genes in the morning glories. The CHS genes are known to comprise two exons with an identical intron position and all the previously characterized Ipomoea CHS genes were reported to bear small introns, ranging in size from 84 to 125 bp (Durbin et al., 1995). The CHS-D and CHS-E genes isolated in this report also consist of two exons with the identical intron position (Fig. 1). However, the sizes of the introns in the CHS-D[M], CHS-D[J], CHS-E[M] and $C H S-E[J]$ are 2,782 bp, 2,680 bp, $338 \mathrm{bp}$ and $352 \mathrm{bp}$, respectively. In young flowers buds, the $C H S-D$ genes were found to be expressed predominantly in the CHS genes (Fukada-Tanaka et al., 1997), and the $C H S-D$ genes contain the longest introns among the known $C H S$ genes in the morning glories (Durbin et al., 1995, this study). In the mutable lines carrying the $a^{\text {flaked }}$ allele for flower variegation, the $C H S-D[\mathrm{M}]$ intron was shown to contain the transposable element Tip100 (Habu et al., 1998). Apart from the Tip100 insertion and its target duplications of $8 \mathrm{bp}$, the $C H S-D[\mathrm{M}]$ gene itself of the mutable line KK/VR-40 is identical to that of the wild-type line KK/FR-35, except that the former carries a T-stretch of $10 \mathrm{bp}$ instead of $9 \mathrm{bp}$ at the position 1545 within the $C H S-D[\mathrm{M}]$ intron (accession number AB004905).

As Fig. 1 shows, homologies between the $C H S-D[\mathrm{M}]$ and $C H S-D[J]$ genes extend not only in exon regions but also within introns and in flanking regions of the genes. Close inspection of the $C H S$ regions revealed the presence of small mobile element-like sequences termed MELS6-1[M], $\triangle M E L S 6-2[\mathrm{M}]$ and MELS6-3 [M] in CHS-D [M] , and MELS3-3[J], $\triangle M E L S 6-1[\mathrm{~J}]$ and MELS6-3[J] in CHS-D[J] (Fig. 2). These MELS elements share structural features with MITEs (miniature inverted-repeat transposable elements) of around 100-400 bp with short terminal inverted repeats (Wessler et al., 1995). Three copies of the newly identified MELS6 elements were also present in the DFR regions of the Japanese and common morning glories, and $\triangle M E L S 6$ sequences are deletion derivatives of MELS6. The MELS3-1 and MELS3-2 elements were previously found in the $D F R$ region of the Japanese morning glory (Inagaki et al., 1999). The three copies of directly repeated sequences of about $193 \mathrm{bp}$ are also present adjacent to the exon 2 in the $C H S-D[\mathrm{M}]$ gene, whereas only one copy of the corresponding sequence of the CHS-D[J] gene is disrupted by an insertion of $529 \mathrm{bp}$ sequence (Fig.
1A). In the stable white flower line of the common morning glory, KK/WP-3, two Tip100 transposons are integrated into the $C H S-D[\mathrm{M}]$ intron and one of them resides within the middle copy of the three tandem repeats of 193 bp (Habu et al., 1998).

Homologous regions between the $C H S-E[\mathrm{M}]$ and $C H S$ $E[\mathrm{~J}]$ genes can be seen in their entire gene sequences including the MELS6-4 elements with only a few break points (Fig. 1B). Two and three copies of small tandem repeats of about $100 \mathrm{bp}$ and $60 \mathrm{bp}$, were detected at the 5 , region and the 3 ' terminus of the CHS-E[J] gene, respectively, and four copies together with an additional truncated copy of long tandem repeats of around $540 \mathrm{bp}$ was found at the right end of the cloned segment. A single copy of the corresponding $60 \mathrm{bp}$ sequence was also found at the 3' terminus of the second exon of the CHS-E[M] gene. Between the CHS-D and CHS-E genes, homologies are restricted to only the exon sequences.

Gene duplication and subsequent divergence are regarded to play important roles in evolution of multiple genes (Ohno, 1970). The phylogenetic tree has indicated that each $C H S[\mathrm{~J}]$ gene is most closely related to the corresponding $C H S[\mathrm{M}]$ gene (Durbin et al., 1995, FukadaTanaka et al., 1997), and the identity between the corresponding $C H S$ genes extended beyond the exon sequences to introns and their flanking regions (Fig. 1). Thus, these results are consistent with gene duplications and major divergence prior to the speciation of the Japanese and common morning glories. Subsequent DNA rearrangements found in Fig. 1 are likely to have taken place after the speciation, and the $M E L S$ sequences appear to play a role in generating such DNA rearrangements.

Expression of the CHS-D and CHS-E genes in the common morning glory. Plants having Tip 100 inserted into the CHS-D[M] gene exhibit variegated pigmentation in flowers, stems and coloring leaves (Imai and Tabuchi, 1935, Epperson and Clegg 1987, Habu et al., 1998), indicating that the $C H S-D[\mathrm{M}]$ gene must be primarily responsible for coloration in these tissues. However, the flower tubes of the CHS-D[M] mutants are often lightly pigmented (Fig. 3). We noticed that, although the pigmentation of their flower tubes in these mutants can vary under different physiological conditions, the mutants from the plants with the purple flowers tend to produce more clear pigmentation in flower tubes than those with red flowers. The observation leads us to suspect that the expression of the CHS-D gene in the young flower buds is predominantly in their flower limbs whereas that of the $C H S-E$ gene is primarily in their flower tubes.

To test the hypothesis, RNAs were prepared from the flower limbs and tubes separately and subjected to Northern blot analysis using the specific probes for the CHS$D[\mathrm{M}]$ or $C H S-E[\mathrm{M}]$ transcripts (Fig. 4). The $C H S-D[\mathrm{M}]$ gene is expressed extensively in the limbs, while the $C H S$ - 


\section{A}

MELS6-1 [M] $\triangle M E L S 6-1[\mathrm{~J}]$ $\triangle$ MELS6-2 [M] MELS6 - 3 [M] MELS6-3 [ J] MELS6 - 4 [M] MELS6-4 [J] MELS6-5 [M] $\triangle$ MELS6-6 [M] MELS6-7 [J]

MELS6-1 [M] $\triangle M E L S 6-1[\mathrm{~J}]$ $\triangle M E L S 6-2[M]$ MELS6 - $3[M]$ MELS6-3 [ U] MELS6-4 [M] MELS6 -4 [J] MELS6-5 [M] $\triangle$ MELS6-6 [M] MELS6-7 [J]

MELS6-1 [M] $\triangle M E L S 6-1[\mathrm{~J}]$ $\triangle M E L S 6-2[M]$ MELS6 -3 [M] MELS6-3 [J] MELS6-4 [M] MELS6-4 [J] MELS6-5 [M] $\triangle M E L S 6-6[M]$ MELS6-7 [J]

MELS6-1 [M] $\triangle$ MELS6-1 [J] $\triangle M E L S 6-2[M]$ MELS6 - 3 [M] MELS6 $-3[\mathrm{~J}]$ MELS6 -4 [M] MELS6-4 [ J] MELS 6-5 [M] $\triangle$ MELS6-6[M] MELS6-7 [J]

B

MELS3 $-3[\mathrm{~J}]$ $\triangle M E L S 3-1[M]$ MELS3-1 [J] MELS3-2 [J]

MELS3-3 [J] $\triangle$ MELS3-1 [M] MELS3 - 1 [J] MELS3 -2 [J]

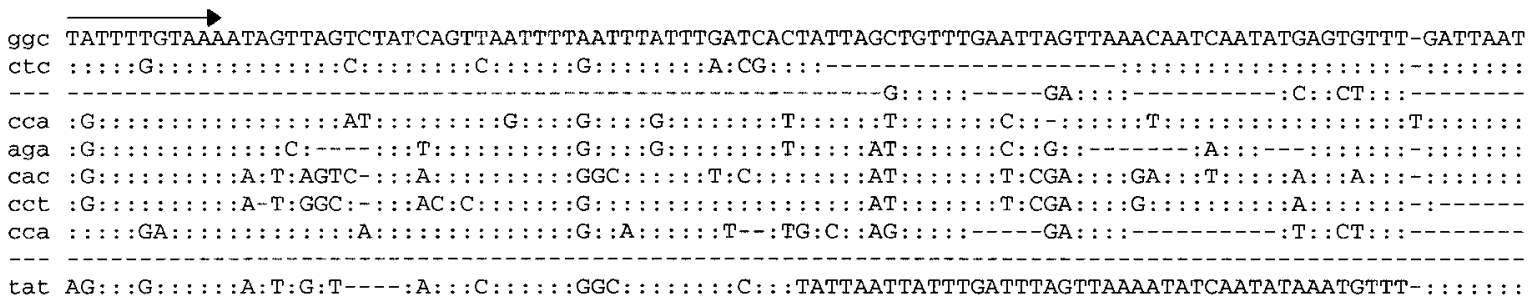

TAACTITTTGTAACAACTTATTGCTCAAAAATA--- TIAAAATTCTAAAAGTTGCTCAAAGTAGCTTTTT-CGATAAACTTTTTGAGAAAGTCATT'T'G : :G:-

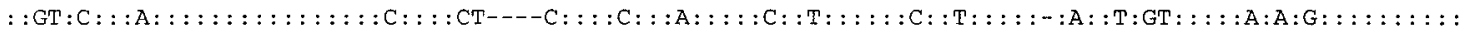

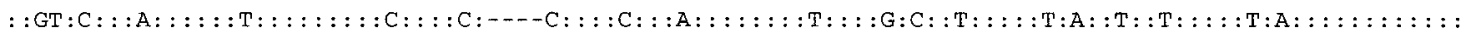

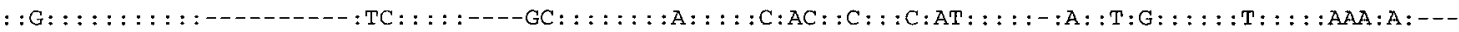

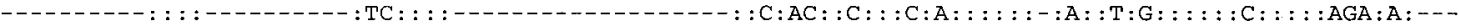
-

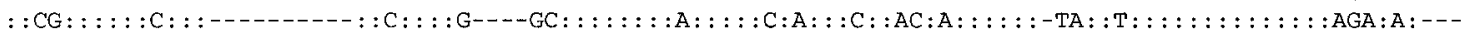

TATGT-AATCATC--TAACAAC----TAACAACTAAT-TTACCAAATACTTT----TCTACAATC--

:

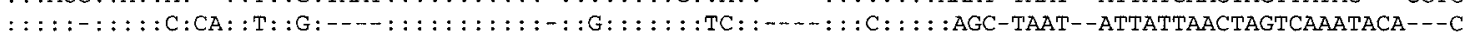

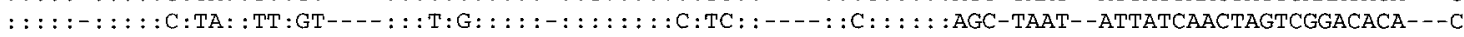
$:::$ ACC $:: A T: G:--:: \mathrm{T}:: \mathrm{G} ;-\cdots--::: \mathrm{T}: \mathrm{G}::::: \mathrm{A}::: \mathrm{G}:::: \mathrm{C}: \mathrm{T}:::---\mathrm{T}: \mathrm{T}-:::: ;-:$ AGC-TAAT--GTTATCAACTAATTATAT---CATC $:::$ ACC $:: A T: G:--:: T:: G:----::::::::::::-:::::::: C:::::---T: A-:$ A $::-:$ AGC-TAAT--GTTATCAACTATTTATAT---CATC

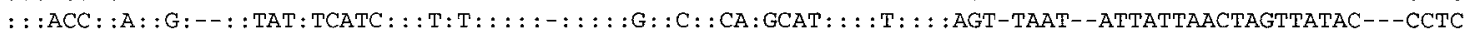

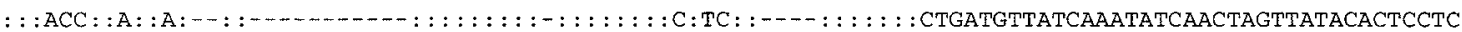

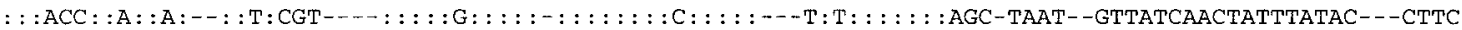

Cat 260

-

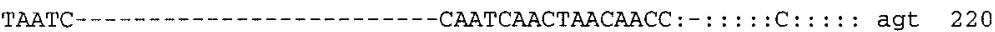

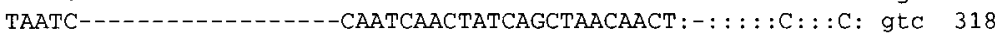

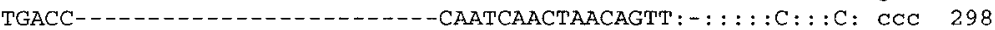

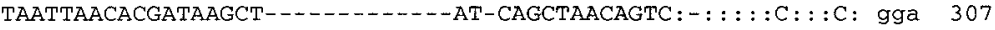

AAATTAATACAATCAGCT-_-...-AA-CAGCTAACCGCC:-: : : : : : : : C: gga 274

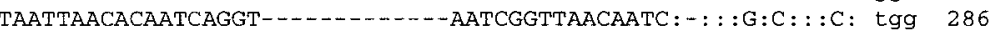

TAATT-

TAATTAACACAATCAGCTAACAGCTAACAACTATTAGCTAACAACC:T:::::C:::C: gga 320

Fig. 2. Structural characteristics of the MELS elements. Alignment of the MELS6 elements (A) and the MELS3 element (B). Nucleotide sequences identical to the upper lines are marked with colons(:) and deleted nucleotides are represented by hyphens (-). The alignment was performed by CLUSTAL W (Thompson et al., 1994). The numerals to the right of the sequences indicate the sizes of the elements and the lower case letters represent the flanking sequences of the elements. The terminal inverted repeats of the MELS6 and MELS3 elements are indicated by the horizontal arrows above the sequences. The MELS6 sequences found in the DFR gene region were taken from DDBJ under the accession numbers AB006793 (DFR[J]) and AB011667 (DFR[M]). MELS6$5[\mathrm{M}], \Delta M E L S 6-6[\mathrm{M}]$ and MELS6-7[J] were found within the first intron of $D F R-A[\mathrm{M}]$, between $D F R-B[\mathrm{M}]$ and $D F R-C[\mathrm{M}]$, and the 5 ' upstream region of DFR-A[J], respectively. MELS3-1 and MELS3-2 in DFR[J] as well as $\triangle M E L S 3-1$ in $D F R[\mathrm{M}]$ were described before (Inagaki et al., 1999).

$E[\mathrm{M}]$ gene is expressed abundantly in the tubes. Interestingly, the $C H S-D[\mathrm{M}]$ mRNAs accumulate in the later stage of the flower development, while the $C H S-E[\mathrm{M}]$ mRNAs accumulate in relatively earlier flower buds (data not shown). The $C H S-D[\mathrm{M}]$ mRNAs were major transcripts in stems, and the $C H S-E[\mathrm{M}]$ gene was found to be induced in sepals after cold treatment (at $15^{\circ} \mathrm{C}$ instead of $25^{\circ} \mathrm{C}$ ) for several days. These results are consistent with the observations that the mutants carrying Tip100 within the $C H S-D$ gene exhibit variegated pigmentation in both corollas and stems and that their sepals become red under low temperature. Among the previously characterized $C H S$ genes in the common morning glory, $C H S-A[\mathrm{M}]$, $C H S-B[\mathrm{M}]$ and $C H S-C[\mathrm{M}]$, both $C H S-A[\mathrm{M}]$ and $C H S-C[\mathrm{M}]$ 


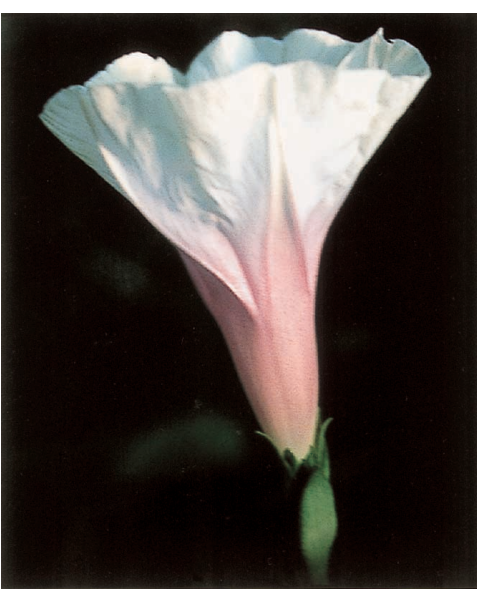

Fig. 3. Flower phenotype of the stable white flower mutant of the common morning glory KK/WP-3 containing two copies of Tip100 within the CHS-D $[\mathrm{M}]$ gene (see Fig. 1A).

were reported to be expressed in the anther and pollen of open flowers (Durbin et al., 1995). We have not examined yet whether $C H S-D[\mathrm{M}]$ or $C H S-E[\mathrm{M}]$ is expressed in these tissues. Future studies should include more detailed examination of the expression patterns of all these CHS genes in various tissues under various environmental stresses such as UV irradiation, cold, heat, drought or wounding. In maize, one of two $\mathrm{CHS}$ genes, $C 2$, is involved in anthocyanin pigmentation in the kernel whereas the other Whp gene is responsible for the CHS activity in pollen (Martin and Gerats 1993).

We have noticed that the $C H S-D[\mathrm{M}]$ gene produces not only the normal mRNAs of about $1.5 \mathrm{~kb}$ but also shorter transcripts of around $1.2 \mathrm{~kb}$ which can be clearly detected in the $C H S-D[\mathrm{M}]$ mutants containing Tip100 (Habu et al., 1997). It is conceivable that the shorter transcripts were produced in the wild-type line KK/FR-35, although they could not have been seen clearly due to abundant production of the normal transcripts. The shorter transcripts are present in the flower limbs as well as in the tubes even in the stable white flower mutant KK/WP-3 carrying two Tip100 elements in the CHS-D[M] intron (Fig. 5). Moreover, the shorter transcripts can be seen in sepals and green leaves where the normal $C H S-D[\mathrm{M}]$ mRNA was scarcely produced (data not shown). To characterize the shorter transcripts further, we prepared four different $C H S-D[\mathrm{M}]$ probes, exon 1 (at the $C H S-D[\mathrm{M}]$ cDNA positions 1-292, see Fukada-Tanaka et al., 1997; this study), 5 ' region of exon 2 (at the positions 307-694), 3' region of exon 2 (at the positions 967-1282) and 3' untranslated region of exon 2 (at the positions 1288-1540, see Fig. 4). Among these probes, the exon 1, 5' region of exon 2 and 3' region of exon 2 were found to be weakly crosshybridizable with the $C H S-E[\mathrm{M}] \mathrm{mRNA}$ of about $1.5 \mathrm{~kb}$ in the lightly pigmented flower tubes of the line KK/WP-3 carrying two copies of Tip100 within the $C H S-D[\mathrm{M}]$ gene (Figs. 1 and 3), while no cross-hybridization was detected
A

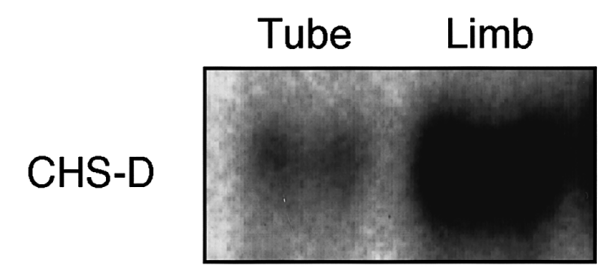

CHS-E

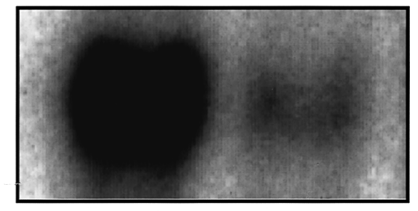

$\gamma$-subunit

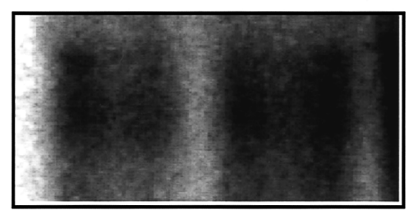

B

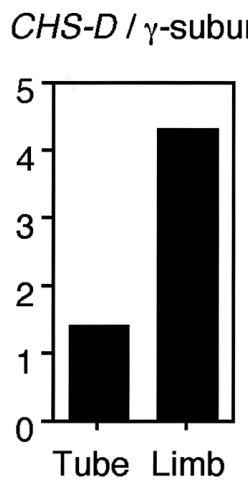

CHS-E / $\gamma$-subunit

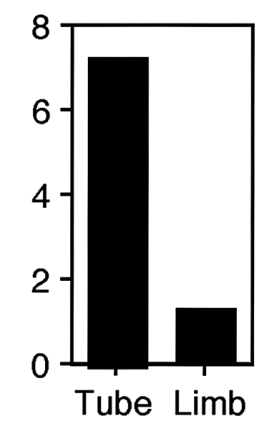

Fig. 4. Expression of the $C H S-D[\mathrm{M}]$ and $C H S-E[\mathrm{M}]$ genes in the common morning glory. Northern blot analysis (A) and production of the $C H S-D[\mathrm{M}]$ and $C H S-E[\mathrm{M}]$ mRNAs relative to the $\gamma$-subunit mRNA of the mitochondrial $\mathrm{F}_{1} \mathrm{~F}_{0}$ ATP synthase (B). Total RNAs $(10 \mu \mathrm{g})$ from flower limbs and tubes of young flower buds of the common morning glory KK/FR-35 were hybridized with the specific $C H S-D[\mathrm{M}]$ and $C H S-D[\mathrm{M}]$ cDNA probes as described by Sambrook et al. (1989). For the specific CHS-D[M] and $C H S-E[\mathrm{M}]$ probes, the 3' untranslated regions of $C H S-D[\mathrm{M}]$ (at the positions 1288 to 1540 ) and $C H S-E[\mathrm{M}]$ (at the positions 1247 to 1392) cDNAs (Fukada-Tanaka et al., 1997) were amplified by the polymerase chain reaction (PCR) with the following primers. Specific $C H S-D[\mathrm{M}]$ probe, YCHSD-2 (5'GCCCGACCCAAATCGAGCCCG-3') and the T7 primer on pBluescript (5'-GTAATACGACTCACTATAGGGC-3'); specific CHS-E[M] probe, SACH-7 (5'-GTTTTGAATTGGGTTTTCATGG3') and SACH-8 (5'-TGTAATGGCTGGCATGGCCTGC-3'). The $\gamma$-subunit cDNA of sweet potato mitochondrial $\mathrm{F}_{1} \mathrm{~F}_{0}$ ATP synthase was used as an internal control (Morikami et al., 1993).

with the probe containing the 3' untranslated region of exon 2. Strong hybridization signals appeared in the white flower limbs of KK/WP-3 when we used these three exon 2 probes but no hybridization was observed with the exon 1 probe (data not shown). These results indicate that the shorter transcripts contain sequence of the $C H S$ - 


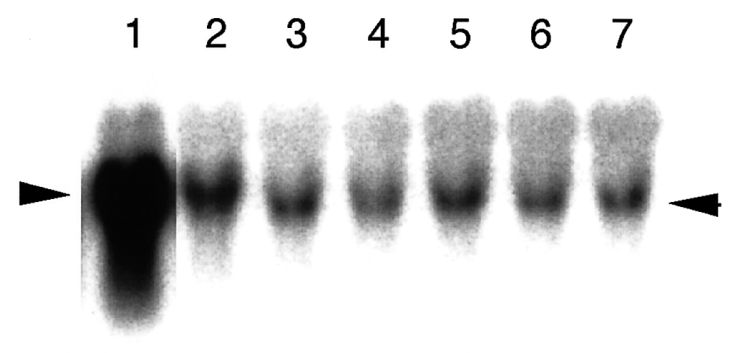

Fig. 5. Production of the shorter $C H S-D[\mathrm{M}]$ transcripts. Northern blot analysis with the specific $C H S-D[\mathrm{M}]$ cDNA probes was performed in the same way as in Fig. 4. Lane 1, flower limbs of fully red flowers from KK/FR-35; lane 2, limbs of heavily variegated flowers from KK/VR-40; lane 3, tubes from KK/VR-40; lane 4 , limbs of lightly variegated flowers from KK/VR-37; lane 5, tubes from KK/VR-37; lane 6, limbs of stable white flowers from KK/ WP-3; lane 7, tubes from KK/WP-3. The mutable lines KK/VR37 and KK/VR-40 carry a single Tip100 at the 5' site whereas the stable white flower line KK/WP-3 contains an additional Tip100 at the 3' site (Fig. 1A). The flower phenotypes of these lines of the common morning glory were shown before (Hisatomi et al., 1997, Habu et al., 1998). Due to heavy variegation, significant amounts of the normal $C H S-D[\mathrm{M}]$ transcripts can be observed in the flower limbs from KK/VR-40. The leftward and rightward arrows point to the normal and shorter $C H S-D[\mathrm{M}]$ transcripts, respectively.

$D[\mathrm{M}]$ second exon including its 3' untranslated region and that the shorter transcripts were produced even in the presence of two copies of Tip100 within the CHS-D[M] intron near exon 1 and exon 2 (Figs. 1 and 5). Indeed, we were able to obtain several cDNA clones containing the exon 2 sequences of the $C H S-D[\mathrm{M}]$ gene from a cDNA library prepared from flower buds of the line KK/VR-37 displaying lightly variegated flowers (Hisatomi et al., 1997, Habu et al., 1998). The regulatory elements for the shorter $C H S-D[\mathrm{M}]$ transcripts as well as their biological significance remain to be elucidated.

According to the classical genetic studies of the Japanese morning glory (Hagiwara 1956, Yoneda and Takenaka 1981), mutations generating white flowers are classified into four groups on the basis of pigmentation on the corolla, flower tube, stem, and seed coat. Among these mutants bearing white flowers, some of them are reported to produce white corollas and pigmented flower tubes with green stems (Hagiwara 1931, Saito et al., 1994). Recently, we have succeeded to identify that one of such mutants indeed carries a mutation in the $\mathrm{CHS}$ $D[\mathrm{~J}]$ gene (A. Hoshino, Y. Johzuka-Hisatomi and S. Iida, unpublished), indicating that the control of expression in the $C H S-D[J]$ and $C H S-E[J]$ genes is similar to that of the $C H S-D[\mathrm{M}]$ and $C H S-E[\mathrm{M}]$ genes. This notion finds support by the sequence similarity at the promoter regions between $C H S-D[\mathrm{~J}]$ and $C H S-D[\mathrm{M}]$ as well as between $C H S-E[\mathrm{M}]$ and $C H S-E[\mathrm{M}]$ (Fig. 1A). It would be intriguing to identify regulatory elements responsible in the promoter regions and the factors acting on these elements, that control the expression patterns of anthocyanin pigmentation in flowers and stems.

The authors thank Miwako Matsumoto for technical assistance, R. Michael Mulligan for critical reading of the manuscript, and Surin Peyachoknagul, Mary Durbin and Michael Clegg for sharing unpublished data. This work is supported in part by grants from the Ministry of Education, Science and Culture, and by the San-Ei Gen Foundation for Food Chemical Research. Y.J.-H. has received research fellowships awarded by the Japan Society for the Promotion of Science for Young Scientists.

\section{REFERENCES}

Durbin, M.L., Learn, G.H., Jr., Huttley, G.A., and Clegg, M.T. (1995) Evolution of the chalcone synthase gene family in the genus Ipomoea. Proc. Natl. Acad. Sci. USA 92, 3338-3342.

Epperson, B.K., and Clegg, M.T. (1987) Instability at a flower color locus in the morning glory. J. Hered. 78, 346-352.

Fukada-Tanaka, S., Hoshino, A., Hisatomi, Y., Habu, Y., Hasebe, M., and Iida, S. (1997) Identification of new chalcone synthase genes for flower pigmentation in the Japanese and common morning glories. Plant Cell Physiol. 38, 754-758.

Habu, Y., Fukada-Tanaka, S., Hisatomi Y., and Iida, S. (1997) Amplified restriction fragment length polymorphism-based mRNA fingerprinting using a single restriction enzyme that recognizes a 4-bp sequence. Biochem. Biophys. Res. Commun. 234, 516-521.

Habu, Y., Hisatomi Y., and Iida, S. (1998) Molecular characterization of the mutable flaked allele for flower variegation in the common morning glory. Plant J. 16, 371-376.

Hagiwara, T. (1931) The genetics of flower colours in Pharbitis Nil. J. Coll. Agric. Imp. Univ. Tokyo 11, 241-262.

Hagiwara, T. (1956) Genes and chromosome maps in the Japanese morning glory. Bull. Res. Coll. Agric. Vet. Sci. Nihon Univ. 5, 34-56.

Harborne, J.B. (1994) The Flavonoids. Advances in Research since 1986. Chapmann and Hall, London.

Hisatomi, Y., Yoneda, Y., Kasahara, K., Inagaki Y., and Iida, S. (1997) DNA rearrangements at the region of the dihydroflavonol 4-reductase gene for flower pigmentation and incomplete dominance in morning glory carrying the mutable flaked mutation. Theor. Appl. Genet. 95, 516-521.

Iida, S., Inagaki, Y., Hisatomi Y., and Hoshino, A. (1995) Mutable alleles in Japanese and common morning glory. In Modification of Gene Expression and Non-Mendelian Inheritance. Edited by Oono K. and Takaiwa, F. pp.23-40. NIAR/STA, Tsukuba.

Imai, Y., and Tabuchi, K. (1935) Recurrent mutation in the flaked alleles of Pharbitis purpurea. J. Genet. 35, 433-446.

Inagaki, Y., Hisatomi, Y., Suzuki, T., Kasahara, K., and Iida, S. (1994) Isolation of a Suppressor-mutator/Enhancer-like transposable element, Tpn1, from Japanese morning glory bearing variegated flowers. Plant Cell 6, 375-383.

Inagaki, Y., Johzuka-Hisatomi, Y., Mori, T., Takahashi, S., Hayakawa, Y., Peyachoknagul, S., Ozeki Y., and Iida, S. (1999) Genomic organization of the genes encoding dihydroflavonol 4-reductase for flower pigmentation in the Japanese and common morning glories. Gene 226, 181-188.

Martin, C.R. (1993) Structure, function, and regulation of the chalcone synthase. International Rev. Cytol. 147, 233-284.

Martin, C. and Gerats, T. (1993) The control of flower coloration. In: The Molecular Biology of Flowering (ed.: B.R. Jordan), pp. 219-255; C.A.B International, Wallingford, Oxon.

Morikami, A., Ehara, G., Yuuki, K., and Nakamura, K. (1993) 
Molecular cloning and characterization of cDNAs for the $\gamma$ and $\varepsilon$-subunits of mitochondorial $\mathrm{F}_{1} \mathrm{~F}_{0}$ ATP synthase from the sweet potato. J. Biol. Chem. 268, 17205-17210.

Ohno, S. (1970) Evolution by Gene Duplication. Springer, New York.

Saito, N., Cheng, C., Ichimura, M., Yokoi, M., Abe, Y., and Honda, T. (1994) Flavonoids in the acyanic flowers of Pharbitis nil. Phytochemistry 35, 687-691.

Sambrook, J., Fritsch, E.F., and Maniatis, T. (1989) Molecular Cloning: a Laboratory Manual. 2nd Ed. Cold Spring Harbor Lab., Cold Spring Harbor, New York.

Shirley, B.W. (1996) Flavonoid biosynthesis: 'new' functions for an 'old' pathway. Trends Plant Sci. 1, 377-382.

Thompson, J.D., Higgins, D.G., and Gibson, T.J. (1994) CLUSTAL $\mathrm{W}$ : improving the sensitivity of progressive multiple sequence alignment through sequence weighting, position-specific gap penalties and weight matrix choice. Nucleic acids Res. 22, 4673-4680.

Wessler, S.R., Bureau, T.E., and White, S.E. (1995) LTRretrotransposons and MITEs: important players in evolution of plant genomes. Curr. Opin. Genet. Devel. 5, 814-821.

Yoneda, Y., and Takenaka, Y. (1981) Colored Illustrations of Japanese Morning Glory (Gensyoku Asagao Kensaku Zukan) (In Japanese). Hokuryukan Publ. Co., Tokyo. 